\title{
Original Article (short paper) \\ Age and sex differences in game-related statistics which discriminate winners from losers in elite basketball games
}

\author{
Haruhiko Madarame ${ }^{1 *}$ \\ ${ }^{1}$ Shigakkan University, Department of Sports and Fitness, Obu, Japan
}

\begin{abstract}
Aims: To advance knowledge of long-term development of basketball players, this study investigated age and sex differences in game-related statistics which discriminate winners from losers in World Basketball Championships held after the 2010 rule change. Methods: A total of 935 games from six categories (under-17, under-19 and open age for both men and women) were analyzed. All games were classified into three types (balanced, unbalanced and very unbalanced) according to point differential by a $k$-means cluster analysis. A discriminant analysis was performed to identify game-related statistics which discriminate winners from losers in each game type. An absolute value of a structural coefficient (SC) equal to or above 0.30 was considered relevant for the discrimination. Results: In balanced games, assists discriminated winners from losers in open games (men, $|\mathrm{SC}|=0.32$; women, $|\mathrm{SC}|=0.34$ ), whereas successful free throws did so in under-17 games (men, $|\mathrm{SC}|=0.30$; women, $|\mathrm{SC}|=0.31$ ). Successful 2-point field goals discriminated winners from losers only in women's games (under-19, $|\mathrm{SC}|=0.38$; open, $|\mathrm{SC}|=0.36$ ). Conclusion: There were three novel findings in balanced games: 1) successful free throws but not assists discriminated winners from losers in under-17 games; 2) successful 2-point field goals discriminated winners from losers in women's games but not in men's games; and 3) discriminating power of successful 3-point field goals was extremely small in women's games. These results may be related to the new rules for the shot clock and the 3-point distance.
\end{abstract}

Keywords: athletes, athletic performance, discriminant analysis, youth sports

\section{Introduction}

Long-term athletic development has drawn a growing interest in recent years. In fact, position statements on the topic have been issued by sports-related organizations such as the International Olympic Committee (IOC) ${ }^{1}$ and the National Strength and Conditioning Association (NSCA) ${ }^{2}$. As youth athletes are physically and mentally immature, it has generally been accepted that training strategies for adult athletes should not be directly applied to youth athletes. Although scientific evidence for long-term athletic development has been accumulated, it is still considered insufficient ${ }^{2}$ especially in the field of team sports ${ }^{3}$, partly because of the complexity of team sports compared to individual sports. Therefore, accumulation of knowledge through scientific studies should be continued in this area.

Basketball is one of the most popular team sports in the world. According to a recent review ${ }^{4}$, topics of basketball studies can be classified into the following fields: shooting performance analysis, individual and team performance analyses using game-related statistics, scoring dynamics, dynamic analysis of space-time coordination and interactions between players and teams, and competitive activity profiles and specific position demands. In the field of statistical studies, a discriminant analysis has been frequently used to identify game-related statistics which discriminate between winning and losing teams in a certain league or tournament ${ }^{5}$. Two of the studies analyzed youth tournaments: 1999 International Basketball Federation (FIBA) Under-18 (U18) World Championship ${ }^{6}$ and 2004 and 2005 FIBA Europe Under-16 (U16) Championships 7 . Although the two studies were well performed, further studies should be required due to several reasons. One of the considerations is the date of tournaments. In 2010, FIBA made significant rule changes which have been reported to affect game-related statistics in toplevel men's tournaments ${ }^{8}$. Therefore, game-related statistics in recent youth tournaments might show different characteristics compared to those in the previously analyzed tournaments. Another important consideration is sex. The two previous studies only analyzed youth tournaments for men. Since the process of biological maturation differs between men and women ${ }^{9}$, the process of development as a basketball player might also differ between men and women. Therefore, to advance knowledge of long-term development of basketball players, this study investigated age and sex differences in game-related statistics which discriminated winners from losers in World Basketball Championships held after the 2010 rule change.

\section{Methods}

\section{Sample and Variables}

Archival data of 935 games were obtained from the official box scores of the FIBA. Competitions included in the data were as follows: $2012(\mathrm{n}=46), 2014(\mathrm{n}=56)$ and $2016(\mathrm{n}=56)$ FIBA Under-17 (U17) World Championships for men; $2011(\mathrm{n}=62)$, $2013(\mathrm{n}=64)$ and $2015(\mathrm{n}=56)$ FIBA Under-19 (U19) World Championships for men; $2012(\mathrm{n}=38)$ Olympic tournament for men, $2014(\mathrm{n}=76)$ FIBA World Championship for men and $2016(n=38)$ Olympic tournament for men; $2012(\mathrm{n}=46), 2014$ $(\mathrm{n}=56)$ and $2016(\mathrm{n}=49)$ FIBA U17 World Championships for women; $2011(\mathrm{n}=57), 2013(\mathrm{n}=63)$ and $2015(\mathrm{n}=56)$ FIBA U19 World Championships for women; $2012(\mathrm{n}=38)$ Olympic tournament for women, $2014(\mathrm{n}=40)$ FIBA World Championship for women and $2016(n=38)$ Olympic tournament for women. 
The following game-related statistics were analyzed: 2 - and 3-point field goals (successful and unsuccessful), free throws (successful and unsuccessful), defensive and offensive rebounds, assists, steals, turnovers, blocks and fouls committed. To eliminate the effect of game rhythm, those variables were normalized to 100 game ball possessions ${ }^{7,10,11}$. Game ball possessions were calculated as an average of team ball possessions (TBP) of both teams. TBP was calculated from field goal attempts (FGA), offensive rebounds (ORB), turnovers (TO) and free throw attempts (FTA) using the following equation ${ }^{12}$ :

$$
\mathrm{TBP}=\mathrm{FGA}-\mathrm{ORB}+\mathrm{TO}+0.4 \times \mathrm{FTA}
$$

\section{Statistical Analysis}

Statistical analyses were performed with $\mathrm{R}$ version 3.3.0 for Windows ${ }^{13}$. All games were first classified into three types (balanced, unbalanced and very unbalanced) according to point differential by a $k$-means cluster analysis ${ }^{7,14,15}$. The classification is shown in Table 1. After the classification, very unbalanced games were eliminated from further analyses ${ }^{14}$. This was because many minutes of very unbalanced games would be "garbage time", and game-related statistics from those minutes have little value for analysis. A three-way ( $\operatorname{sex} \times$ age $\times$ game result) analysis of variance (ANOVA) was performed to assess differences and interactions in each variable. A discriminant analysis was performed using R code 'candis' (http://aoki2.si.gunma-u. ac.jp/R/src/candis.R) and 'geneig' (http://aoki2.si.gunma-u. ac.jp/R/src/geneig. R) to identify game-related statistics which discriminate winners from losers in each game type. An absolute value of a structural coefficient (SC) equal to or above 0.30 was considered relevant for the discrimination ${ }^{7,11,16}$. Statistical significance was set at $\mathrm{P}<0.05$.

Table 1. Game classification by final point differentials

\begin{tabular}{|c|c|c|c|}
\hline & Balanced & Unbalanced & Very unbalanced \\
\hline Point differential & $\leq 16$ & 17 to 39 & $\geq 40$ \\
\hline \multicolumn{4}{|l|}{ Number (\%) } \\
\hline U17 Men & $83(53)$ & $48(30)$ & $27(17)$ \\
\hline U19 Men & $114(63)$ & $48(26)$ & $20(11)$ \\
\hline Open Men & $89(59)$ & $54(36)$ & $9(6)$ \\
\hline U17 Women & $80(53)$ & $59(39)$ & $12(8)$ \\
\hline U19 Women & $83(47)$ & $75(43)$ & $18(10)$ \\
\hline Open Women & $71(61)$ & $33(28)$ & $12(10)$ \\
\hline
\end{tabular}

\section{Results}

Normalized game-related statistics and results of a three-way ANOVA are shown in Table 2 (balanced games) and Table 3 (unbalanced games). In balanced games, there were significant sex $\times$ age interactions for successful 2-point field goals $[F(2$, $1028)=8.73, P<0.001]$, unsuccessful 2-point field goals $[F$ $(2,1028)=5.78, P<0.01]$, successful 3-point field goals $[F(2$, $1028)=5.11, P<0.01]$, blocks $[F(2,1028)=4.55, P<0.05]$ and fouls committed $[F(2,1028)=5.11, P<0.01]$. Significant sex $\times$ game result interactions were observed for successful 2-point field goals $[F(1,1028)=8.04, P<0.01]$ and 3 -point field goals $[F(1$, $1028)=4.54, P<0.05]$. For variables without any interaction, main effects of three factors were all significant except for assists, where main effects of two factors (age and game results) were significant [age, $F(2,1028)=111.84, P<0.01$; game results, $F(1,1028)=61.39, P<0.01]$. In unbalanced games, there was a significant sex $\times$ age $\times$ game result interaction for offensive rebounds $[F(2,622)=4.26, P<0.05]$, and an age $\times$ game result interaction for assists $[F(2,622)=5.62, P<0.01]$. In addition, there were significant sex $\times$ age interactions for successful 2-point field goals $[F(2,622)=6.03, P<0.01]$, unsuccessful 2-point field goals $[F(2,622)=4.76, P<0.01]$, and unsuccessful 3-point field goals $[F(2,622)=3.85, P<0.05]$, and sex $\times$ game result interactions for successful 2-point field goals $[F(1,622)=6.29$,
$P<0.05]$, successful 3-point field goals $[F(1,622)=11.20, P$ $<0.001]$ and fouls committed $[F(1,622)=4.20, P<0.05]$. For variables without any interaction, main effects of three factors were significant for defensive rebounds [sex, $F(1,622)=5.52, P$ $<0.05$; age, $F(2,622)=4.77, P<0.01$; game result, $F(1,622)=$ $430.13, P<0.001$ ], steals [sex, $F(1,622)=28.81, P<0.001$; age, $F(2,622)=32.65, P<0.001$; game result, $F(1,622)=90.15, P$ $<0.001]$ and turnovers [sex, $F(1,622)=49.58, P<0.001$; age, $F(2,622)=39.70, P<0.001$; game result, $F(1,622)=47.38, P$ $<0.001]$. Results of a discriminant analysis are shown in Table 4 and Figure 1. In balanced games, assists discriminated winners from losers in open games (men, $|\mathrm{SC}|=0.32$; women, $|\mathrm{SC}|=0.34$ ), whereas successful free throws did so in U17 games (men, $|\mathrm{SC}|=$ 0.30 ; women, $|\mathrm{SC}|=0.31$ ). In addition, winners were discriminated from losers by successful 2-point field goals (U19 women, $|\mathrm{SC}|$ $=0.38$; open women, $|\mathrm{SC}|=0.36$ ) and defensive rebounds (U19 men, $|\mathrm{SC}|=0.35$; open men, $|\mathrm{SC}|=0.31$; U17 women, $|\mathrm{SC}|=$ 0.31 ; U19 women, $|\mathrm{SC}|=0.33$ ). In unbalanced games, winners were discriminated from losers by successful 2-point field goals (U17 men, $|\mathrm{SC}|=0.32$; U17 women, $|\mathrm{SC}|=0.42$; U19 women, $|\mathrm{SC}|=0.40$; open women, $|\mathrm{SC}|=0.33$ ), defensive rebounds (U17 men, $|\mathrm{SC}|=0.30$; U19 men, $|\mathrm{SC}|=0.31$; U17 women, $|\mathrm{SC}|=0.35$; U19 women, $|\mathrm{SC}|=0.34$; open women, $|\mathrm{SC}|=0.33$ ) and assists (open men, $|\mathrm{SC}|=0.32$; U17 women, $|\mathrm{SC}|=0.39$; U19 women, $|\mathrm{SC}|=0.30$; open women, $\mathrm{SC} \mid=0.37$ ). 
Table 2. Game-related statistics of balanced games

\begin{tabular}{|c|c|c|c|c|c|c|c|c|c|c|c|c|c|}
\hline & & \multicolumn{6}{|c|}{ Men } & \multicolumn{6}{|c|}{ Women } \\
\hline & & \multicolumn{2}{|c|}{ U17 } & \multicolumn{2}{|c|}{ U19 } & \multicolumn{2}{|c|}{ Open } & \multicolumn{2}{|c|}{ U17 } & \multicolumn{2}{|c|}{ U19 } & \multicolumn{2}{|c|}{ Open } \\
\hline & & $\mathbf{W}$ & $\mathbf{L}$ & $\mathbf{W}$ & $\mathbf{L}$ & $\mathbf{W}$ & $\mathbf{L}$ & $\mathbf{W}$ & $\mathbf{L}$ & $\mathbf{W}$ & $\mathbf{L}$ & $\mathbf{W}$ & $\mathbf{L}$ \\
\hline \multirow[t]{2}{*}{ S2P $P^{a, b, c, d, e}$} & Mean & 26.8 & 25.0 & 28.1 & 25.6 & 27.9 & 26.7 & 23.8 & 21.8 & 28.7 & 24.1 & 29.9 & 25.5 \\
\hline & SD & 6.7 & 6.4 & 6.9 & 5.7 & 5.4 & 5.2 & 5.7 & 5.7 & 5.8 & 4.7 & 6.1 & 5.6 \\
\hline \multirow[t]{2}{*}{$\mathrm{U} 2 \mathrm{P}^{\mathrm{a}, \mathrm{b}, \mathrm{c}, \mathrm{d}}$} & Mean & 31.4 & 32.5 & 29.3 & 31.7 & 26.0 & 28.1 & 39.5 & 38.9 & 36.1 & 35.3 & 35.4 & 37.5 \\
\hline & SD & 8.4 & 7.1 & 8.8 & 8.3 & 6.5 & 6.5 & 9.1 & 8.6 & 9.4 & 7.9 & 8.0 & 8.0 \\
\hline \multirow[t]{2}{*}{$\mathrm{S} 3 \mathrm{P}^{\mathrm{a}, \mathrm{b}, \mathrm{c}, \mathrm{d}, \mathrm{e}}$} & Mean & 8.0 & 7.4 & 9.0 & 8.1 & 11.3 & 9.8 & 5.8 & 5.2 & 6.3 & 6.5 & 7.2 & 6.9 \\
\hline & SD & 4.3 & 3.9 & 4.3 & 4.4 & 3.9 & 3.8 & 3.4 & 3.3 & 3.2 & 3.6 & 3.0 & 3.3 \\
\hline \multirow[t]{2}{*}{$\mathrm{U} 3 \mathrm{P}^{\mathrm{a}, \mathrm{b}, \mathrm{c}}$} & Mean & 19.8 & 20.8 & 18.3 & 20.5 & 19.4 & 20.4 & 15.6 & 17.4 & 14.7 & 18.5 & 14.5 & 15.6 \\
\hline & SD & 6.4 & 6.4 & 5.9 & 6.4 & 5.3 & 5.8 & 6.5 & 8.0 & 6.7 & 6.2 & 5.5 & 4.7 \\
\hline \multirow[t]{2}{*}{$\mathrm{SFT}^{\mathrm{a}, \mathrm{b}, \mathrm{c}}$} & Mean & 19.6 & 15.4 & 19.5 & 16.6 & 20.7 & 18.1 & 18.4 & 14.0 & 17.5 & 15.2 & 18.2 & 16.1 \\
\hline & SD & 6.9 & 6.3 & 7.0 & 6.5 & 7.2 & 7.9 & 7.6 & 5.8 & 6.5 & 7.0 & 6.6 & 6.1 \\
\hline \multirow[t]{2}{*}{$\mathrm{UFT}^{\mathrm{a}, \mathrm{b}, \mathrm{c}}$} & Mean & 9.3 & 9.3 & 9.1 & 8.9 & 8.3 & 6.8 & 9.2 & 7.7 & 8.7 & 8.0 & 6.3 & 5.8 \\
\hline & SD & 5.5 & 5.2 & 4.2 & 4.0 & 4.5 & 3.9 & 5.4 & 5.2 & 5.0 & 5.2 & 3.2 & 3.2 \\
\hline \multirow[t]{2}{*}{$\mathrm{DRB}^{\mathrm{a}, \mathrm{b}, \mathrm{c}}$} & Mean & 38.7 & 35.8 & 35.9 & 31.8 & 36.4 & 32.9 & 39.5 & 36.0 & 38.1 & 33.4 & 37.8 & 34.5 \\
\hline & SD & 7.0 & 7.1 & 6.3 & 6.0 & 6.6 & 5.6 & 7.6 & 7.0 & 6.4 & 6.0 & 6.2 & 5.5 \\
\hline \multirow[t]{2}{*}{$\mathrm{ORB}^{\mathrm{a}, \mathrm{b}, \mathrm{c}}$} & Mean & 18.9 & 17.9 & 16.2 & 16.6 & 15.1 & 13.5 & 21.8 & 19.1 & 18.7 & 16.6 & 17.1 & 16.8 \\
\hline & SD & 7.0 & 6.0 & 7.3 & 6.2 & 5.3 & 4.8 & 7.4 & 6.6 & 7.6 & 5.9 & 6.0 & 6.6 \\
\hline \multirow[t]{2}{*}{$\mathrm{AST}^{\mathrm{b}, \mathrm{c}}$} & Mean & 16.4 & 14.5 & 18.8 & 16.3 & 23.4 & 19.9 & 14.5 & 13.0 & 19.0 & 15.7 & 24.3 & 20.1 \\
\hline & $\mathrm{SD}$ & 6.1 & 5.3 & 6.0 & 6.0 & 6.5 & 5.7 & 5.7 & 4.5 & 6.4 & 5.4 & 6.1 & 6.0 \\
\hline \multirow[t]{2}{*}{$\mathrm{STL}^{\mathrm{a}, \mathrm{b}, \mathrm{c}}$} & Mean & 12.3 & 10.4 & 10.9 & 9.7 & 8.9 & 8.3 & 15.1 & 13.3 & 12.5 & 11.3 & 10.5 & 8.7 \\
\hline & SD & 4.7 & 3.9 & 4.2 & 3.7 & 3.8 & 3.4 & 5.2 & 4.3 & 4.7 & 4.6 & 4.5 & 3.7 \\
\hline \multirow[t]{2}{*}{$\mathrm{TO}^{\mathrm{a}, \mathrm{b}, \mathrm{c}}$} & Mean & 21.2 & 22.5 & 20.1 & 20.6 & 18.6 & 18.9 & 25.7 & 27.5 & 22.6 & 22.7 & 20.4 & 22.4 \\
\hline & SD & 5.1 & 6.3 & 5.4 & 5.4 & 4.4 & 5.3 & 6.1 & 7.7 & 5.5 & 6.4 & 5.5 & 5.9 \\
\hline \multirow[t]{2}{*}{$\mathrm{BLK}^{\mathrm{b}, \mathrm{c}, \mathrm{d}}$} & Mean & 4.6 & 4.5 & 4.4 & 3.6 & 4.0 & 3.1 & 5.1 & 3.6 & 4.0 & 3.4 & 5.2 & 3.8 \\
\hline & SD & 2.9 & 3.5 & 2.8 & 2.5 & 2.6 & 2.4 & 3.7 & 2.4 & 2.8 & 2.7 & 3.5 & 2.5 \\
\hline \multirow[t]{2}{*}{$\mathrm{FC}^{\mathrm{a}, \mathrm{b}, \mathrm{c}, \mathrm{d}}$} & Mean & 25.2 & 26.8 & 26.7 & 28.5 & 28.6 & 30.3 & 23.2 & 25.5 & 23.7 & 25.3 & 25.3 & 26.4 \\
\hline & SD & 6.0 & 5.5 & 5.7 & 5.4 & 5.5 & 4.9 & 5.6 & 5.9 & 6.4 & 4.9 & 4.4 & 5.5 \\
\hline
\end{tabular}

W, winners; L, losers; S2P, successful 2-point field goals; U2P, unsuccessful 2-point field goals; S3P, successful 3-point field goals; U3P, unsuccessful 3-point field goals; SFT, successful free throws; UFT, unsuccessful free throws; DRB, defensive rebounds; ORB, offensive rebounds; AST, assists; STL, steals; TO, turnovers; BLK, blocks; FC, fouls committed. a, main effect of sex; b, main effect of age; c, main effect of game result; $d$, sex $\times$ age interaction; e, sex $\times$ game result interaction $(\mathrm{P}<0.05)$.

Table 3. Game-related statistics of unbalanced games

\begin{tabular}{|c|c|c|c|c|c|c|c|c|c|c|c|c|c|}
\hline & & \multicolumn{6}{|c|}{ Men } & \multicolumn{6}{|c|}{ Women } \\
\hline & & \multicolumn{2}{|c|}{ U17 } & \multicolumn{2}{|c|}{ U19 } & \multicolumn{2}{|c|}{ Open } & \multicolumn{2}{|c|}{ U17 } & \multicolumn{2}{|c|}{ U19 } & \multicolumn{2}{|c|}{ Open } \\
\hline & & $\mathbf{W}$ & $\mathbf{L}$ & $\mathbf{W}$ & $\mathbf{L}$ & $\mathbf{W}$ & $\mathbf{L}$ & $\mathbf{W}$ & $\mathbf{L}$ & $\mathbf{W}$ & $\mathbf{L}$ & $\mathbf{W}$ & $\mathbf{L}$ \\
\hline \multirow[t]{2}{*}{$\mathrm{S} 2 \mathrm{P}^{\mathrm{a}, \mathrm{b}, \mathrm{c}, \mathrm{d}, \mathrm{e}}$} & Mean & 31.1 & 21.6 & 33.3 & 22.6 & 33.0 & 24.8 & 28.6 & 16.8 & 30.6 & 19.1 & 35.3 & 23.2 \\
\hline & SD & 6.3 & 4.2 & 7.8 & 5.5 & 5.2 & 4.5 & 6.8 & 5.6 & 5.9 & 6.0 & 6.7 & 5.0 \\
\hline \multirow[t]{2}{*}{$\mathrm{U} 2 \mathrm{P}^{\mathrm{a}, \mathrm{b}, \mathrm{c}, \mathrm{d}}$} & Mean & 31.2 & 33.5 & 29.1 & 36.1 & 24.8 & 31.2 & 36.1 & 39.6 & 34.0 & 37.2 & 34.0 & 38.3 \\
\hline & SD & 9.1 & 8.5 & 7.5 & 8.5 & 6.6 & 8.0 & 6.6 & 8.6 & 9.3 & 8.6 & 7.8 & 6.8 \\
\hline \multirow[t]{2}{*}{$\mathrm{S} 3 \mathrm{P}^{\mathrm{a}, \mathrm{b}, \mathrm{c}, \mathrm{e}}$} & Mean & 8.7 & 6.1 & 8.8 & 6.3 & 12.4 & 7.3 & 6.5 & 4.8 & 7.2 & 5.7 & 8.1 & 6.3 \\
\hline & SD & 3.7 & 3.8 & 3.7 & 3.1 & 4.6 & 3.3 & 3.2 & 2.6 & 3.7 & 3.0 & 3.8 & 3.1 \\
\hline \multirow[t]{2}{*}{$\mathrm{U} 3 \mathrm{P}^{\mathrm{a}, \mathrm{c}, \mathrm{d}}$} & Mean & 18.0 & 20.3 & 17.1 & 20.3 & 19.2 & 19.1 & 14.8 & 20.4 & 16.0 & 18.5 & 13.4 & 15.4 \\
\hline & SD & 6.9 & 7.3 & 6.3 & 6.4 & 5.3 & 6.2 & 6.8 & 7.5 & 6.4 & 6.3 & 5.7 & 4.7 \\
\hline \multirow[t]{2}{*}{$\mathrm{SFT}^{\mathrm{a}, \mathrm{c}}$} & Mean & 18.5 & 14.0 & 19.0 & 14.7 & 19.2 & 16.0 & 18.4 & 12.1 & 17.9 & 12.9 & 18.4 & 13.9 \\
\hline & $\mathrm{SD}$ & 6.7 & 6.0 & 7.7 & 6.9 & 6.7 & 7.2 & 6.7 & 6.5 & 7.8 & 5.6 & 8.4 & 5.6 \\
\hline
\end{tabular}




\begin{tabular}{|c|c|c|c|c|c|c|c|c|c|c|c|c|c|}
\hline \multirow[t]{2}{*}{$\mathrm{UFT}^{\mathrm{b}, \mathrm{c}}$} & Mean & 10.5 & 9.5 & 9.8 & 7.2 & 7.0 & 7.0 & 10.0 & 9.0 & 8.7 & 7.2 & 6.0 & 6.0 \\
\hline & SD & 5.8 & 5.4 & 4.6 & 3.9 & 3.4 & 3.6 & 5.2 & 5.1 & 4.1 & 4.6 & 4.1 & 4.3 \\
\hline \multirow[t]{2}{*}{$\mathrm{DRB}^{\mathrm{a}, \mathrm{b}, \mathrm{c}}$} & Mean & 41.7 & 29.8 & 40.4 & 28.3 & 38.7 & 30.2 & 43.7 & 31.3 & 40.8 & 29.8 & 41.3 & 29.7 \\
\hline & SD & 7.8 & 6.3 & 7.5 & 6.8 & 5.8 & 5.8 & 9.4 & 5.9 & 7.3 & 6.1 & 5.2 & 6.3 \\
\hline \multirow[t]{2}{*}{$\mathrm{ORB}^{\mathrm{a}, \mathrm{b}, \mathrm{c}, \mathrm{g}}$} & Mean & 23.9 & 15.7 & 19.3 & 15.2 & 16.4 & 13.0 & 22.3 & 18.7 & 20.5 & 14.2 & 19.2 & 14.6 \\
\hline & SD & 7.2 & 5.6 & 6.0 & 5.6 & 6.1 & 6.3 & 7.1 & 6.2 & 7.2 & 7.0 & 6.6 & 6.7 \\
\hline \multirow[t]{2}{*}{$\mathrm{AST}^{\mathrm{a}, \mathrm{b}, \mathrm{c}, \mathrm{f}}$} & Mean & 21.3 & 12.8 & 23.6 & 14.6 & 28.5 & 16.7 & 19.0 & 10.6 & 20.7 & 12.5 & 28.5 & 16.5 \\
\hline & SD & 5.4 & 5.2 & 6.6 & 5.3 & 6.3 & 5.8 & 5.6 & 3.8 & 6.6 & 4.7 & 5.7 & 4.8 \\
\hline \multirow[t]{2}{*}{$\mathrm{STL}^{\mathrm{a}, \mathrm{b}, \mathrm{c}}$} & Mean & 13.8 & 10.8 & 11.8 & 9.4 & 11.0 & 7.4 & 15.6 & 12.5 & 15.3 & 10.4 & 10.6 & 8.0 \\
\hline & SD & 4.5 & 4.3 & 4.3 & 3.8 & 4.4 & 4.1 & 6.2 & 4.7 & 5.2 & 4.2 & 3.6 & 3.6 \\
\hline \multirow[t]{2}{*}{$\mathrm{TO}^{\mathrm{a}, \mathrm{b}, \mathrm{c}}$} & Mean & 23.5 & 24.7 & 19.6 & 21.2 & 16.4 & 21.4 & 25.1 & 28.5 & 22.1 & 25.8 & 18.9 & 23.3 \\
\hline & SD & 5.8 & 7.1 & 6.1 & 5.2 & 5.3 & 5.4 & 5.8 & 7.6 & 5.5 & 6.0 & 5.4 & 5.4 \\
\hline \multirow[t]{2}{*}{$\mathrm{BLK}^{\mathrm{a}, \mathrm{c}}$} & Mean & 4.9 & 4.0 & 5.6 & 3.8 & 4.9 & 3.8 & 5.3 & 3.5 & 4.3 & 2.4 & 4.8 & 2.9 \\
\hline & SD & 2.8 & 2.5 & 3.3 & 1.9 & 3.4 & 3.0 & 3.9 & 2.9 & 3.3 & 2.2 & 3.4 & 2.1 \\
\hline \multirow[t]{2}{*}{$\mathrm{FC}^{\mathrm{a}, \mathrm{b}, \mathrm{c}, \mathrm{e}}$} & Mean & 24.9 & 26.6 & 24.3 & 26.5 & 26.7 & 28.2 & 21.7 & 26.3 & 20.9 & 24.5 & 23.1 & 25.2 \\
\hline & SD & 6.4 & 5.5 & 6.2 & 6.2 & 6.0 & 5.4 & 5.6 & 5.9 & 5.0 & 6.7 & 6.3 & 6.5 \\
\hline
\end{tabular}

W, winners; L, losers; S2P, successful 2-point field goals; U2P, unsuccessful 2-point field goals; S3P, successful 3-point field goals; U3P, unsuccessful 3-point field goals; SFT, successful free throws; UFT, unsuccessful free throws; DRB, defensive rebounds; ORB, offensive rebounds; AST, assists; STL, steals; TO, turnovers; BLK, blocks; $F C$, fouls committed. a, main effect of sex; $b$, main effect of age; $c$, main effect of game result; $d$, sex $\times$ age interaction; e, sex $\times$ game result interaction; $\mathrm{f}$, age $\times$ game result interaction; $\mathrm{g}$, sex $\times$ age $\times$ game result interaction $(\mathrm{P}<0.05)$.

Table 4. Results of discriminant analysis with structural coefficients (SC) of game-related statistics

\begin{tabular}{|c|c|c|c|c|c|c|c|c|c|c|c|c|}
\hline & \multicolumn{6}{|c|}{ Balanced } & \multicolumn{6}{|c|}{ Unbalanced } \\
\hline & \multicolumn{3}{|c|}{ Men } & \multicolumn{3}{|c|}{ Women } & \multicolumn{3}{|c|}{ Men } & \multicolumn{3}{|c|}{ Women } \\
\hline & U17 & U19 & Open & U17 & U19 & Open & U17 & U19 & Open & U17 & U19 & Open \\
\hline EV & 1.13 & 0.91 & 0.83 & 1.21 & 1.31 & 1.06 & 7.89 & 7.93 & 9.24 & 5.17 & 5.90 & 9.69 \\
\hline WL & 0.47 & 0.52 & 0.55 & 0.45 & 0.43 & 0.49 & 0.11 & 0.11 & 0.10 & 0.16 & 0.14 & 0.09 \\
\hline $\mathrm{CS}$ & 119.1 & 142.0 & 102.8 & 119.9 & 131.9 & 96.5 & 191.2 & 191.6 & 231.5 & 199.3 & 273.4 & 136.2 \\
\hline $\mathrm{P}$ & $<0.01$ & $<0.01$ & $<0.01$ & $<0.01$ & $<0.01$ & $<0.01$ & $<0.01$ & $<0.01$ & $<0.01$ & $<0.01$ & $<0.01$ & $<0.01$ \\
\hline $\mathrm{CC}$ & 0.73 & 0.69 & 0.67 & 0.74 & 0.75 & 0.72 & 0.94 & 0.94 & 0.95 & 0.92 & 0.92 & 0.95 \\
\hline $\mathrm{RC}$ & $83 \%$ & $84 \%$ & $83 \%$ & $86 \%$ & $86 \%$ & $83 \%$ & $100 \%$ & $100 \%$ & $100 \%$ & $97 \%$ & $100 \%$ & $100 \%$ \\
\hline $\mathrm{S} 2 \mathrm{P}$ & 0.13 & 0.20 & 0.13 & 0.24 & $-0.38 *$ & $-0.36^{*}$ & $-0.32 *$ & 0.28 & 0.28 & $-0.42 *$ & $0.40^{*}$ & $-0.33^{*}$ \\
\hline U2P & -0.06 & -0.15 & -0.18 & 0.02 & -0.04 & 0.12 & 0.05 & -0.16 & -0.14 & 0.10 & -0.07 & 0.10 \\
\hline S3P & 0.07 & 0.11 & 0.21 & -0.02 & 0.02 & -0.04 & -0.13 & 0.13 & 0.21 & -0.13 & 0.09 & -0.09 \\
\hline U3P & -0.08 & -0.19 & -0.11 & -0.19 & 0.26 & 0.11 & 0.06 & -0.09 & 0.00 & 0.17 & -0.08 & 0.06 \\
\hline SFT & $0.30^{*}$ & 0.22 & 0.19 & $0.31^{*}$ & -0.15 & -0.16 & -0.12 & 0.11 & 0.07 & -0.21 & 0.16 & -0.10 \\
\hline UFT & 0.00 & 0.02 & 0.19 & 0.06 & -0.06 & -0.08 & -0.03 & 0.11 & 0.00 & -0.04 & 0.07 & 0.00 \\
\hline DRB & 0.20 & $0.35^{*}$ & $0.31^{*}$ & $0.31^{*}$ & $-0.33 *$ & -0.27 & $-0.30^{*}$ & $0.31^{*}$ & 0.25 & $-0.35^{*}$ & $0.34^{*}$ & $-0.33 *$ \\
\hline ORB & 0.08 & -0.03 & 0.18 & 0.18 & -0.13 & -0.02 & -0.23 & 0.13 & 0.09 & -0.12 & 0.19 & -0.11 \\
\hline AST & 0.16 & 0.22 & $0.32 *$ & 0.16 & -0.24 & $-0.34 *$ & -0.29 & 0.27 & $0.32 *$ & $-0.39 *$ & $0.30^{*}$ & $-0.37 *$ \\
\hline STL & 0.21 & 0.16 & 0.09 & 0.10 & -0.11 & -0.21 & -0.12 & 0.11 & 0.14 & -0.12 & 0.21 & -0.12 \\
\hline TO & -0.11 & -0.06 & -0.04 & -0.01 & 0.01 & 0.17 & 0.03 & -0.05 & -0.16 & 0.11 & -0.13 & 0.13 \\
\hline BLK & 0.01 & 0.14 & 0.20 & 0.25 & -0.10 & -0.23 & -0.06 & 0.12 & 0.05 & -0.12 & 0.15 & -0.11 \\
\hline $\mathrm{FC}$ & -0.13 & -0.18 & -0.18 & -0.18 & 0.13 & 0.11 & 0.05 & -0.06 & -0.04 & 0.18 & -0.13 & 0.05 \\
\hline
\end{tabular}

EV, eigenvalue; WL, Wilks' Lambda; CS, chi-square; CC, canonical correlation; RC, reclassification; S2P, successful 2-point field goals; U2P, unsuccessful 2-point field goals; S3P, successful 3-point field goals; U3P, unsuccessful 3-point field goals; SFT, successful free throws; UFT, unsuccessful free throws; DRB, defensive rebounds; ORB, offensive rebounds; AST, assists; STL, steals; TO, turnovers; BLK, blocks; FC, fouls committed. * $|\mathrm{SC}| \geq 0.30$. 
Figure 1. Structural coefficients (SC) of game-related statistics in balanced (A) and unbalanced (B) games. S2P, successful 2-point field goals; U2P, unsuccessful 2-point field goals; S3P, successful 3-point field goals; U3P, unsuccessful 3-point field goals; SFT, successful free throws; UFT, unsuccessful free throws; DRB, defensive rebounds; ORB, offensive rebounds; AST, assists; STL, steals; TO, turnovers; BLK, blocks; FC, fouls committed; M, men; W, women. $|\mathrm{SC}| \geq 0.30$ was considered relevant for discrimination.
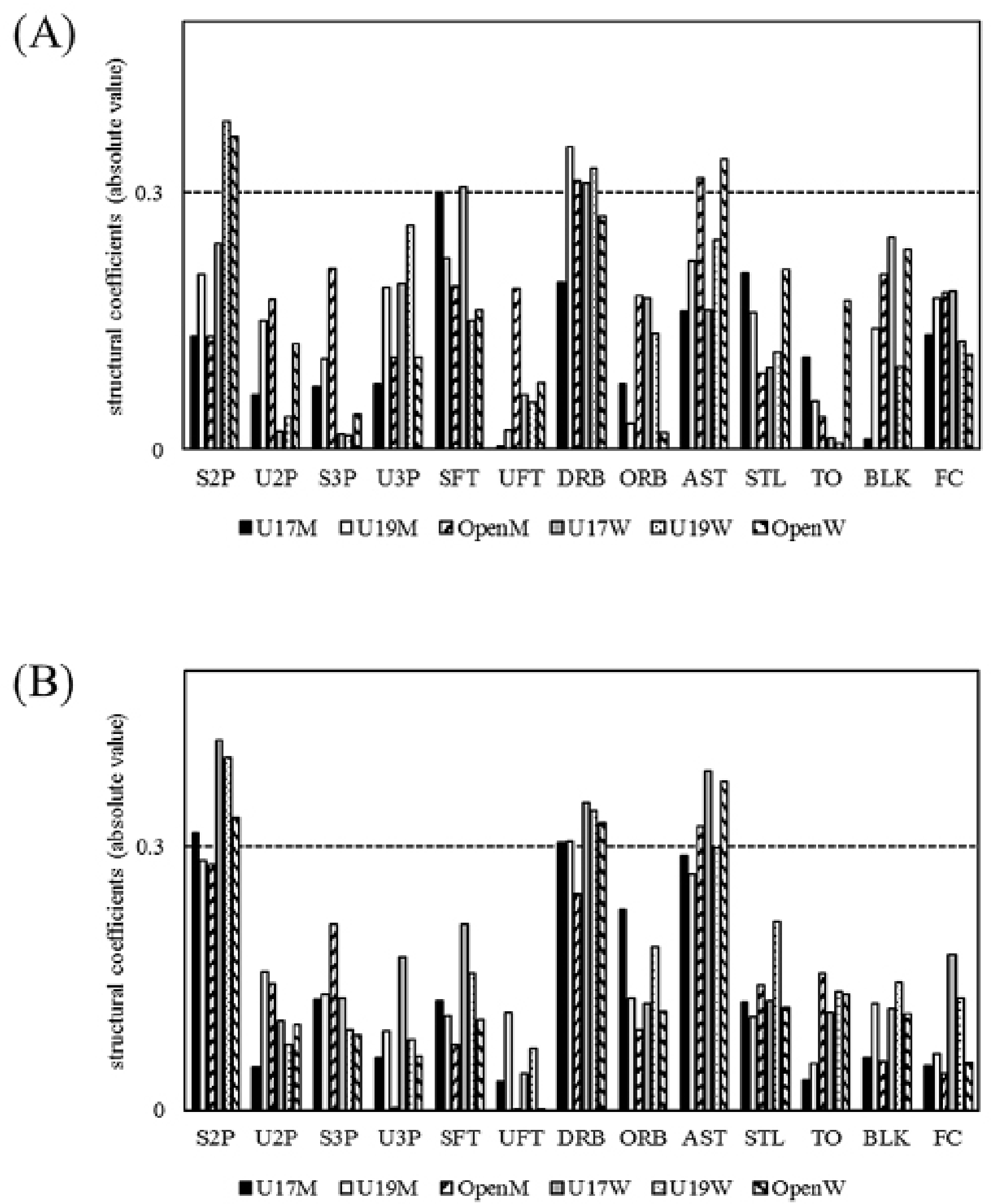


\section{Discussion}

To advance knowledge of long-term development of basketball players, this study investigated age and sex differences in gamerelated statistics which discriminated winners from losers in World Basketball Championships held after the 2010 rule change. There were three novel findings in balanced games: 1) successful free throws but not assists discriminated winners from losers in U17 games; 2) successful 2-point field goals discriminated winners from losers in women's games but not in men's games; and 3) discriminating power of successful 3-point field goals was extremely small in women's games.

In balanced games, assists discriminated winners from losers in open games, whereas successful free throws did so in U17 games. These results were observed for both men and women, and the average number of assists per 100 game ball possessions also increased with age, independent of sex. Assists have been reported as a discriminant factor between winners and losers, and have been considered as an indicator of player's perceptual and decision-making abilities ${ }^{7,14,17}$. It is likely that due to players' immature in perception and decision-making, organized and efficient offenses were less in U17 than in open games, and thus U17 games were discriminated by free throws rather than field goals. In contrast to the present study, Lorenzo and colleagues ${ }^{7}$ reported that assists discriminated winners from losers in close games (point differences equal to or below nine points) in 2004 and 2005 FIBA Europe U16 Championships for men $(|\mathrm{SC}|=$ $0.33)$. This discrepancy may be due to changes in FIBA rules. Under the new rules which have been effective since 2010, the shot clock is not reset to 24 seconds in certain situations, forcing the offensive team to attempt a field goal in a short time. As this situation requires more advanced abilities in perception and decision-making, it may be difficult for youth teams to adjust.

In balanced games, although winners in women's U19 and open games were discriminated from losers in those categories by successful 2-point field goals, men's games were not discriminated by them. These results are in line with recent studies on Asian and European Championships ${ }^{18,19}$. It seems to be a recent trend that women's balanced games are discriminated by successful 2-point field goals, whereas men's balanced games are not. In contrast to 2-point field goals, although the absolute values of SC did not exceed 0.30 , the values for successful 3-point field goals were larger in men's games than in women's games in all age categories. The values in women's games were extremely small. This result was different from a previous study by Gomez and colleagues ${ }^{10}$, reporting that successful 3-point field goals discriminated winners from losers in balanced games in 2004 and 2005 Spanish women's league $(|\mathrm{SC}|=0.37)$. This discrepancy may also be explained by one of the rule changes in 2010, in which the 3-point distance has been extended from $6.25 \mathrm{~m}$ to $6.75 \mathrm{~m}$. As women are generally inferior to men in muscle strength, women would be largely affected by the increase in the 3-point distance.

In unbalanced games, winners were discriminated from losers mostly by successful 2-point field goals, defensive rebounds and assists. Although the absolute values of SC for those three variables were below 0.30 in some categories, all the values exceeded 0.25 , and the largest three values in each category were from those three variables. These results are consistent with previous studies reporting that successful 2-point field goals ${ }^{7,10,11,18}$, defensive rebounds ${ }^{7,10,11,18}$ and assists ${ }^{11,14,18}$ discriminate winners from losers in unbalanced games. It seems that, independent of age and sex, securing a defensive rebound to gain ball possession and running well-organized offense to make an easy basket are essential for winning a game by a huge margin.

Limitations of this study should be acknowledged. Although analysis on game-related statistics is suitable for quantitatively assessing large amounts of data, it is not enough to fully investigate the game of basketball. This limitation can be compensated by studies on specific elements of the game such as screen ${ }^{20,21,22}$, foul $^{23,24}$ and substitution ${ }^{25}$.

Practical implications for long-term development of basketball players can be drawn from this study. In balanced games, assists, which have been considered as an indicator of player's perceptual and decision-making abilities, discriminated winners from losers in open games, whereas successful free throws did so in U17 games. Simply looking at the results, we can say that improving free throw shooting would increase the number of winning games in youth tournaments. However, just focusing on immediate wins would not lead to long-term player development. As the new shot clock rule forces the offensive team to attempt a field goal in a short time, coaches should put emphasis on developing perceptual and decision-making abilities of youth players so that they can succeed in the future.

\section{Conclusion}

This study showed that in balanced games, 1) successful free throws but not assists discriminated winners from losers in U17 games; 2) successful 2-point field goals discriminated winners from losers in women's games but not in men's games; and 3) discriminating power of successful 3-point field goals was extremely small in women's games. These results may be related to the new rules for the shot clock and the 3-point distance.

\section{References}

1. Bergeron MF, Mountjoy M, Armstrong N, Chia M, Cote J, Emery CA, et al. International Olympic Committee consensus statement on youth athletic development. Br J Sports Med. 2015;49(13):843-51.

2. Lloyd RS, Cronin JB, Faigenbaum AD, Haff GG, Howard $\mathrm{R}$, Kraemer WJ, et al. National Strength and Conditioning Association Position Statement on Long-Term Athletic Development. J Strength Cond Res. 2016;30(6):1491-509.

3. Burgess DJ, Naughton GA. Talent development in adolescent team sports: a review. Int J Sports Physiol Perform. 2010;5(1):103-16.

4. Volossovitch A. Research Topics in Basketball. In: Passos P, Araújo D, Volossovitch A, editors. Performance Analysis in Team Sports. Oxfordshire and New York: Routledge; 2017. pp. 173-99.

5. Sampaio J, Ibáñez SJ, Lorenzo A. Basketball. In: McGarry T, O’Donoghue P, Sampaio J, editors. Routledge Handbook of Sports 
Performance Analysis. Oxfordshire and New York: Routledge; 2013. pp. 357-66.

6. Ibáñez SJ, Sampaio J, Saenz-Lopez P, GImenez J, Janeira MA. Game statistics discriminating the final outcome of Junior World Basketball Championship matches (Portugal 1999). J Hum Mov Stud. 2003;45:1-19.

7. Lorenzo A, Gómez MA, Ortega E, Ibáñez SJ, Sampaio J. Game related statistics which discriminate between winning and losing under-16 male basketball games. J Sports Sci Med. 2010;9(4):664-8.

8. Strumbelj E, Vracar P, Robnik-Sikonja M, Dezman B, Erculj F. A decade of euroleague basketball: an analysis of trends and recent rule change effects. J Hum Kinet. 2013;38:183-9.

9. Lloyd RS, Oliver JL, Faigenbaum AD, Myer GD, De Ste Croix MB. Chronological age vs. biological maturation: implications for exercise programming in youth. J Strength Cond Res. 2014;28(5):1454-64.

10. Gómez MA, Lorenzo A, Sampaio J, Ibáñez SJ. Differences in game-related statistics between winning and losing teams in women's basketball. J Hum Mov Stud. 2006;51(5):357-69.

11. Gómez MA, Lorenzo A, Sampaio J, Ibáñez SJ, Ortega E. Gamerelated statistics that discriminated winning and losing teams from the Spanish men's professional basketball teams. Coll Antropol. 2008;32(2):451-6.

12. Oliver D. Watching a game: Offensive score sheets. Basketball on paper: Rules and tools for performance analysis. Washington D.C.: Potomac Books; 2004. pp. 8-28.

13. R Core Team. R: A language and environment for statistical computing. Vienna, Austria: R Foundation for Statistical Computing; 2016.

14. Garcia J, Ibáñez SJ, De Santos RM, Leite N, Sampaio J. Identifying basketball performance indicators in regular season and playoff games. J Hum Kinet. 2013;36:161-8.

15. Csataljay G, O’Donoghue P, Hughes M, Dancs H. Performance indicators that distinguish winning and losing teams in basketball. Int J Perform Anal Sport. 2009;9(1):60-6.

16. Gómez MA, Lorenzo A, Barakat R, Ortega E, Palao JM. Differences in game-related statistics of basketball performance by game location for men's winning and losing teams. Percept Mot Skills. 2008;106(1):43-50.

17. Puente C, Coso JD, Salinero JJ, Abián-Vicén J. Basketball performance indicators during the ACB regular season from 2003 to 2013. Int J Perform Anal Sport. 2015;15(3):935-48.
18. Madarame H. Game-related statistics which discriminate between winning and losing teams in Asian and European men's basketball championships. Asian J Sports Med. 2017;8(2):e42727.

19. Madarame H. Defensive rebounds discriminate winners from losers in European but not in Asian women's basketball championships. Asian J Sports Med. in press.

20. Gómez MA, Battaglia O, Lorenzo A, Lorenzo J, Jimenez S, Sampaio J. Effectiveness during ball screens in elite basketball games. J Sports Sci. 2015;33(17):1844-52.

21. Marmarinos C, Apostolidis N, Kostopoulos N, Apostolidis A. Efficacy of the "pick and roll" offense in top level European basketball teams. J Hum Kinet. 2016;51:121-9.

22. Vaquera A, García-Tormo JV, Ruano M.A G, Morante JC. An exploration of ball screen effectiveness on elite basketball teams. Int J Perform Anal Sport. 2016;16(2):475-85.

23. Gómez MA, Ortega E, Jones G. Investigation of the impact of 'fouling out' on teams' performance in elite basketball. Int J Perform Anal Sports. 2016;16(3):983-94.

24. Gómez MA, Ortega Toro E, Furley P. The Influence of Unsportsmanlike Fouls on Basketball Teams' Performance According to Context-Related Variables. Int J Sports Physiol Perform. 2016;11(5):664-70.

25. Gómez MA, Silva R, Lorenzo A, Kreivyte R, Sampaio J. Exploring the effects of substituting basketball players in highlevel teams. J Sports Sci. 2017;35(3):247-54.

\section{Corresponding author}

*Haruhiko Madarame

Department of Sports and Fitness, Shigakkan University, Nakoyama 55, Yokonemachi, Obu, Aichi 474-8651, Japan

Email: madarame-tky@umin.ac.jp

Manuscript received on November 1, 2017

Manuscript accepted on December 13, 2017

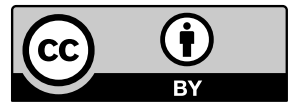

Motriz. The Journal of Physical Education. UNESP. Rio Claro, SP, Brazil - eISSN: 1980-6574 - under a license Creative Commons - Version 4.0 